\title{
A multichannel 3D cell-based biosensor for real-time and non-invasive monitoring of 3D cell proliferation and drug screening
}

\author{
Yuxiang Pan ${ }^{1,3}$, Xinwei Wei ${ }^{1}$, Hao Wan ${ }^{1}$, Ning Hu ${ }^{2,3^{*}}$, Ping Wang ${ }^{1,3,{ }^{*}}$ \\ ${ }^{1}$ Biosensor National Special Laboratory, Key Laboratory for Biomedical Engineering of Education \\ Ministry, Department of Biomedical Engineering, Zhejiang University, Hangzhou 310027, China; \\ ${ }^{2}$ Harvard-Massachusetts Institute of Technology Division of Health Sciences and Technology, \\ Cambridge, MA 02139 \\ ${ }^{3}$ State Key Laboratory of Transducer Technology, Chinese Academy of Sciences, Shanghai, 200050, \\ China \\ *Corresponding Tel/Fax: +86 571 87952832, Email: cnpwang@zju.edu.cn
}

\begin{abstract}
:
A multichannel 3D electric cell-substrate impedance sensing assay has been developed for real-time and non-invasive monitoring of 3D cell proliferation and drug susceptibility. In this study, human liver cancer cells (HepG2) was encapsulated in 3D matrigel and cultured in a miniaturized chamber. 3D cell culture systems represent more accurately the actual microenvironment where cells reside in tissues. A pair of vertical golden electrodes were embedded at the opposite sidewalls of the culture chamber for the on-site impedance measurement. Cell density in the 3D construct was shown to be proportional to the impedance magnitude of the entire construct. The homemade portable 8-channel ECIS system was used for long-time noninvasively monitoring the impedance of cells. Moreover, when the cell proliferation reached stagnate phase, real-time impedimetric monitoring of cell viability under different anti-cancer drugs was conducted and the impedance magnitude was directly correlated with the cell viability. The experimental results showed that cell proliferation and drug susceptibility in 3D cell culture model can be monitored by this 3D ECIS system. This 3D cell-based biosensor has a high potential to develop a powerful analytical platform for cancer research and drug screening.
\end{abstract}

Key words: 3D cell culture, Multichannel biosensor, Impedance measurement, Cell proliferation, Drug screening

\section{Introduction}

Three-dimensional (3D) cell culture system have gained increasing interest in cancer research and drug screening due to their evident advantages in stimulating the complex in vivo microenvironments. Currently, in drug discovery, the standard procedure of screening compounds starts with the 2D cell culturebased tests, followed by animal model tests, to clinical trials. Cell-based biosensors have become an important pillar of the drug discovery process by serving as a simple, fast, and cost-effective alternative to large-scale and cost-intensive animal testing. However, 2D cell culture may not adequately represent the natural context of the cell and results in less accurate predictions of cellular response to the drug. As such, 3D-cultured cells can be the ideal sensing element in cell-based sensors to provide the most biologically relevant information and predictive data for in vivo tests.
However, the conventional techniques for monitoring 3D cell proliferation and viability are normally labor-intensive and time-consuming. A real time, high throughput and label free method for detection of $3 \mathrm{D}$ cell proliferation is in urgent need.

\section{Methods}

HepG2 cells suspension mixed with matrigel were seeded into chambers of 8 channel 3D ECIS chip and recording cell impedance real time. Drug test conducted by Gemcitabine (GCB, 10mM) and Paclitaxel (TAX, 1mM). As for data analysis, cell index $(\mathrm{Cl})$ is defined as:

$$
C I=\left|\frac{Z_{\text {cell }}-Z_{\text {baseline }}}{Z_{\text {baseline }}}\right|
$$

\section{Results and Discussion}

Cell density in the 3D construct was shown to be proportional to the impedance magnitude of the entire construct. When cells are electrically 
stimulated, gap junctions of the cells effectively connect electrically to the cells. As a result, the sensitivity of cells by externally applying electrical field increases and cells may act as a conductor for a short time by considerable biochemical and biophysical changes (Fig. 1A). The detection system of 3D ECIS contains signal generator module, amplification and filter module, and data acquisition module. A pair of vertical electrodes were embedded at the opposite sidewalls of the culture chamber for the on-site impedance measurement (Fig. 1B, C).

A

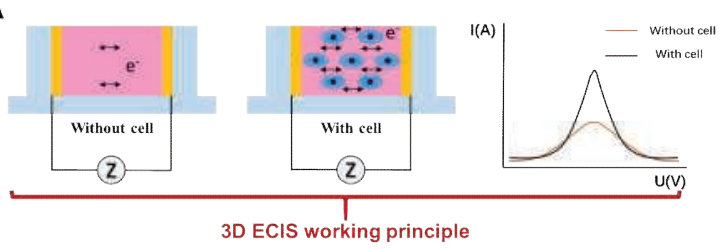

B

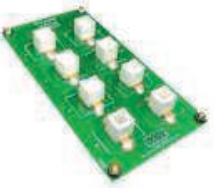

C

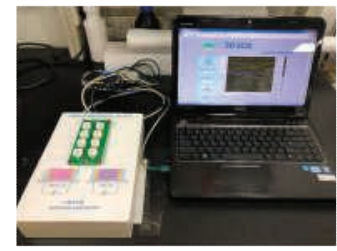

Fig. 1 A. Schematic of $3 D$ cell-based biosensor. B. 8 channel 3D ECIS chip. C. 3D ECIS system.

The Bode plot of the 3D cell construct was showed in Fig. 2A and compared with the construct without cells. At the frequency of 10 $\mathrm{kHz}$, the impedance magnitude presents the most significant difference. We also tested the stability of 3D ECIS system used blank matrigel without cell (Fig. 2B).
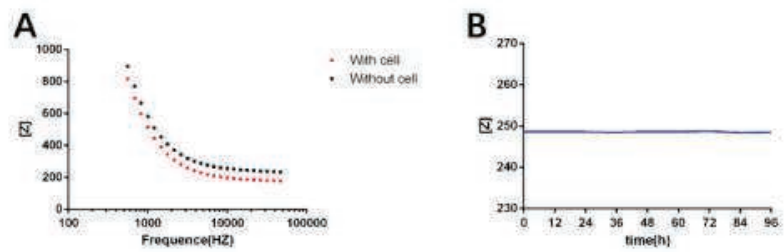

Fig. 2. A. Bode plot of the cells/matrigel construct and the construct without cells, B. Stability of the $3 D$ cell-based biosensor detection system.

Human liver cancer cells (HepG2) was selected to establish the 3D cell-based biosensor. Fig. 3 showed the morphology and viability of $3 D$ cultured HepG2 cells. Fig. 4A showed the cell growth curves of 3D HepG2 cells measured by 3D ECIS system with different cell numbers ranging from 10000 to 50,000 cells/well. Based on the obtained results and analysis, 40,000 was the suitable seeding number to obtain a better cell-based biosensor. Fig. 4B showed a group of normalized cell growth curves of HepG2 cells responding to TAX and GCB treatment.

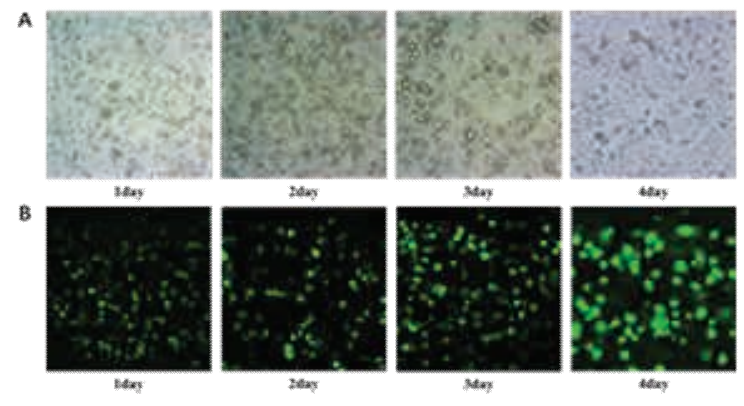

Fig.3 A. Morphology 3D cultured HepG2 cell observed by optical microscope. B. Live cell staining (CalceinAM) of long-time cultured 3D HepG2 cell.

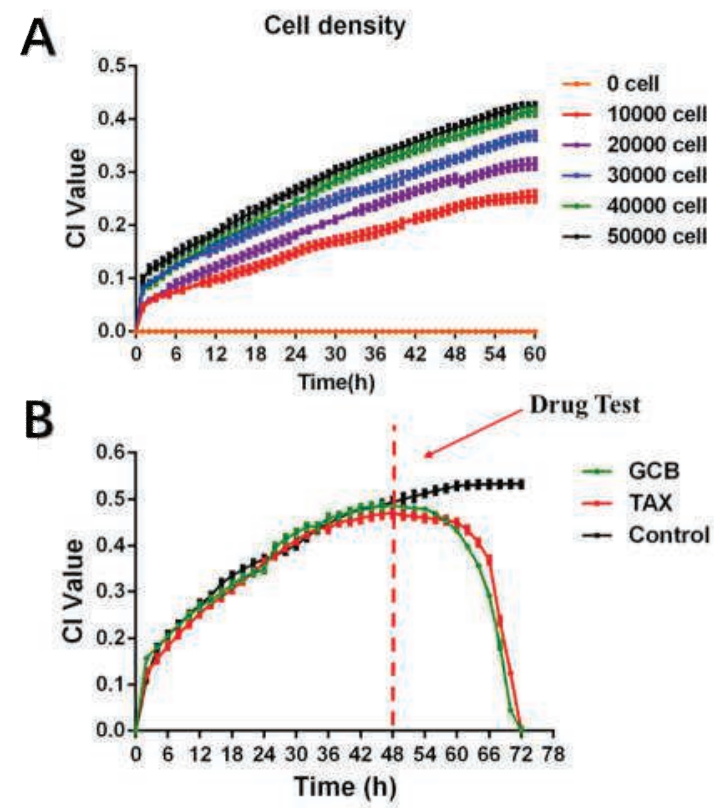

Fig. 4 A.Cell growth curves of HepG2 cells at different cell numbers. B. Drug test

\section{Conclusion}

Our work provide a useful means to observe 3D cell responses in real time. The ultimate goal is to use the proposed system to assay the sensitivities of patient's cancer cells to anticancer drugs at 3D tumor tissue or 3D cell culture level. In future research, we will use more drugs to test the stability of the platform and apply the platform to improve the accuracy of drug screening.

\section{References}

[1] Lei, Kin Fong, et al. "Real-time and non-invasive impedimetric monitoring of cell proliferation and chemosensitivity in a perfusion 3D cell culture microfluidic chip." Biosensors and Bioelectronics 51 (2014): 16-21.Doi: 10.1016/j.bios.2013.07.031

[2] Edmondson, Rasheena, et al. "Three-dimensional cell culture systems and their applications in drug discovery and cell-based biosensors." Assay and drug development technologies 12.4 (2014): 207-218. Doi: 10.1089/adt.2014.573 